\title{
Evaluation of the efficacy of octreotide LAR in the treatment of Crohn's disease associated refractory diarrhea
}

\author{
Laura Martelli $^{\mathrm{a}}$, Arnaud Colard ${ }^{\mathrm{a}}$, Fernand Fontaine ${ }^{\mathrm{a}}$, Jacques Deflandre ${ }^{\mathrm{b}}$, Boris Bastens ${ }^{\mathrm{a}}$ and Edouard \\ Louis $^{\mathrm{C}}$
}

${ }^{a}$ Department of Gastroenterology, CHC Clinique Saint-Joseph, Liège, Belgium; ${ }^{b}$ Department of Gastroenterology, CHR Citadelle, Liège, Belgium; 'Department of Gastroenterology, University Hospital CHU of Liège, Liège, Belgium

\begin{abstract}
Objectives: Diarrhea is one of the main symptoms of Crohn's disease (CD). It is usually significantly improved with specific CD treatments, loperamide or cholestyramine. However, in some cases, diarrhea becomes refractory. The aim of this study was to assess the safety and efficacy of octreotide in this situation.
\end{abstract}

Materials and methods: Fifteen patients with CD refractory diarrhea defined by at least an average of five smooth or liquid stools per day despite an optimized CD treatment were included from three Belgian centers. Two patients were lost to follow-up. A subcutaneous injection of $100 \mu \mathrm{g}$ octreotide was performed three times a day during three days. When the drug had been well tolerated, an intramuscular injection of $30 \mathrm{mg}$ octreotide (Sandostatin ${ }^{\circledR}$ LAR 30) was realized. Evaluation was done at day 31. The primary endpoint was to assess the effect on the mean number of smooth or liquid stools per day.

Results: A significant reduction ( $p=0.0001)$ of the average number of smooth or liquid stools over the last seven days was observed between baseline and day 31. The maximum number of smooth or liquid stools also significantly decreased $(p=0.0009)$. Four patients $(26.7 \%)$ presented mild nonspecific adverse events but no serious one. We also observed a significant decrease $(p=0.0006)$ of the Harvey-Bradshaw Index (HBI) and a significant improvement $(p=0.0012)$ of the inflammatory bowel disease questionnaire (IBDQ).

Conclusions: In this uncontrolled open-label study, octreotide appeared safe and effective in CD refractory diarrhea, in addition to $C D$ treatments. It significantly improved the number of liquid or smooth stools, the HBI and the IBDQ.

\section{KEYWORDS}

Crohn's disease; octreotide; open-label study; refractory diarrhea; Sandostatin® LAR

\section{Introduction}

Diarrhea is one of the main symptoms of Crohn's disease (CD). Its mechanisms are complex and multifactorial. Malabsorption, osmotic mechanisms, secretory, and motility anomalies may be involved. Diarrhea is usually significantly improved by specific CD treatments including steroids, immunosuppressive drugs, and biologics. Loperamide and cholestyramine may also be useful. However, in some cases, diarrhea persists, and becomes refractory because of an incomplete control of the intestinal inflammatory reaction despite an optimization of $C D$ treatment or due to persisting functional diarrhea despite a complete control of the intestinal inflammatory reaction. In these situations, octreotide, through its effects on the gastrointestinal tract secretion and motility [1-3] and its potential immunomodulatory effect [4] may be useful. Octreotide is indeed already used in some cases of $C D$ associated refractory diarrhea although no clear evidence of its efficacy is available in the literature. The aim of this study was thus to assess the safety and efficacy of octreotide in CD refractory diarrhea, in addition to CD treatments.

\section{Materials and methods}

The protocol was approved by the Ethics committee of Liège University Hospital in June 2011. Before undergoing any study assessment, patients gave their written informed consent. 
Published in: Laura Martelli, Arnaud Colard, Fernand Fontaine, Jacques Deflandre, Boris Bastens \& Edouard Louis (2017) Evaluation of the efficacy of octreotide LAR in the treatment of Crohn's disease associated refractory diarrhea, Scandinavian Journal of Gastroenterology, 52:5, 564-569, DOI: 10.1080/00365521.2017.1284893

Status: Postprint (author's version)

CD patients, according to Lennard-Jones criteria [5] were prospectively recruited from three Belgian regional and University centers: Center Hospitalier Chrétien CHC Liège, Center Hospitalier Régional de la Citadelle CHR Liège, University Hospital CHU Liège.

\section{Study design}

To be included in the study, the CD patients had to be aged eighteen or older, and to present refractory diarrhea. Refractory diarrhea was defined by at least a mean of five smooth or liquid stools per day (mean of the number of stools per day over the last seven days prior to baseline) despite a normal C-reactive protein (CRP) and an optimized specific CD treatment including immunosuppressive drugs and/or anti-TNF. We used the Bristol Stool Scale [6] to evaluate the consistency of the stools. Diarrhea had also to be refractory to loperamide and/or cholestyramine. To be considered refractory to these drugs, the patients had to have insufficient control of the diarrhea according to their own assessment, with either $8 \times 42 \mathrm{mg}$ of loperamide and/or $2 \times 4 \mathrm{~g}$ of cholestyramine. They were also considered refractory if they could not tolerate these drugs. Patients with previous surgical resection could be included in the study provided that the intervention had been done at least six months before the inclusion in the trial.

Patients could not be included in the study if they presented a known allergy or intolerance to octreotide. They were also excluded if they had diarrhea due to small bowel microbial proliferation, lactose intolerance, irradiation, chemotherapy, neoplasia, or ischemia. Small bowel microbial proliferation was excluded by either a breath test or by an absence of improvement after a course of antibiotics. In addition, the inclusion in the study was not allowed if they presented an immediate need for surgery, a significant dehydration with the need for intravenous saline perfusion or a severe malnutrition with a Body Mass Index $(\mathrm{BMI})<16$ with the need for enteral or parenteral nutrition. Finally, a Harvey-Bradshaw Index (HBI) [7]; $>13$ or a sub score for pain or general wellbeing $>2$ were also exclusion criteria.

Mesalazine was allowed at a stable dosage for at least three months. Methylprednisolone was tolerated at a dosage below $24 \mathrm{mg}$ and stable for two weeks while budesonide was tolerated at a dosage equal or below $6 \mathrm{mg}$ and stable for two weeks. Azathioprine, 6-mercaptopurine, and methotrexate were permitted at a stable dosage for at least three months. Infliximab treatment was approved, but day 0 of the present study had to correspond to day 15 to day 28 after the last perfusion, and no new perfusion could be performed during the 31 days of the study. Antibiotics for $C D$ were allowed at a stable dosage for at least two weeks while antibiotics for another indication were only permitted for a short-course of treatment. Loperamide therapy was allowed at a maximum dose of $3 \times 2 \mathrm{mg} / \mathrm{d}$ and had to remain stable throughout the study while cholestyramine was permitted at a maximum dosage of $2 \times 4 \mathrm{~g} / \mathrm{d}$ and had also to remain stable throughout the study.

\section{Treatment evaluated during the study}

A subcutaneous injection of $100 \mu \mathrm{g}$ of octreotide (Sandostatin ${ }^{\circledR} 100 \mu \mathrm{g} / \mathrm{ml}$ s.c.) was performed three times a day during the first three days after baseline. When the drug had been well tolerated, an intramuscular injection of $30 \mathrm{mg}$ of octreotide (Sandostatin® LAR 30) was realized on day 3.

Patients discontinued the trial in case of any CD treatment change, including any change in the dosage of $C D$ treatment, $C D$ surgery, and intolerance or study treatment side-effects.

\section{Endpoints}

The primary endpoints were to assess the safety and the effect of subcutaneous octreotide $100 \mu \mathrm{g}$ three times a day for three days followed by one intramuscular injection of octreotide $30 \mathrm{mg}$ LAR on $C D$ refractory diarrhea. Evaluation was done at day 31. The criterion of judgment was the mean number of smooth or liquid stools per day by comparing the last seven days before baseline and before day 31 (stool frequency being collected by a patient on a daily basis all over the study period in a diary).

As secondary endpoint, we evaluated the effect of octreotide therapy on the mean number and on the maximum number of bowel movements per day between baseline and day 31 . We also assessed the number of patients responding to octreotide. A response was defined by a decrease of at least $25 \%$ of the mean number of liquid or smooth stools per day between baseline and day 31 . The proportion of patients with a significant reduction in the number of liquid or smooth stools per day, defined by a reduction of at least 2, was also assessed. Moreover, we assessed the effect of octreotide on CD activity by calculating the $\mathrm{HBI}$, on CRP (measured locally on day 0 and 31 ), on fecal calprotectin 
Published in: Laura Martelli, Arnaud Colard, Fernand Fontaine, Jacques Deflandre, Boris Bastens \& Edouard Louis (2017) Evaluation of the efficacy of octreotide LAR in the treatment of Crohn's disease associated refractory diarrhea, Scandinavian Journal of Gastroenterology, 52:5, 564-569, DOI: 10.1080/00365521.2017.1284893

Status: Postprint (author's version)

(measured centrally at CHU Liège, ELISA Bühlmann) and on health related quality of life Inflammatory bowel Disease Questionnaire (IBDQ) [8].

\section{Statistical analysis}

The primary endpoint was to assess the effect of octreotide on the mean number of smooth or liquid stools per day. These mean numbers were compared between the seven days preceding day 0 and day 31 in the thirteen patients for whom we had these two measurements by a paired f-test (two patients were lost to follow-up). An intent-to-treat analysis was performed considering patients lost to follow-up as non-responders and keeping the same number of stools as at baseline.

The evolution of the other parameters included in the secondary endpoints (total number of bowel movements, $\mathrm{HBI}, \mathrm{CRP}, \mathrm{IBDQ}$, and fecal calprotectin) was assessed in the same way. Demographic and clinical characteristics were compared between responders and non-responders.

Results were presented as means, standard deviations (SD), medians, and interquartile ranges (IQR) for continuous variables and as tables of frequencies for categorical variables. Absolute differences (between day 0 and day 31) and relative differences ([day 0-day 31] x 100/day 0) were also evaluated.

Results were considered significant if the $p$ value was inferior to 0.05 . The statistical analysis was done using the software SAS version 9.4. ${ }^{* * *}$

\section{Results}

\section{Description of the study population}

Fifteen patients, $8(53.3 \%)$ women and $7(46.7 \%)$ men, with a median age of 51.2 years (IQR: $39.7-$ 58.0 years) were included. They were recruited in the three participating centers ( 10 at $\mathrm{CHC}, 3$ at $\mathrm{CHU}$ and 2 at CHR). The characteristics of the patients are described in Table 1. Two patients were lost to follow-up and could not be assessed for efficacy. Those were considered as non-responders and their data at baseline were used for response assessment.

\section{Impact on the number of smooth or liquid stools between day 0 and day 31}

The mean number of smooth or liquid stools significantly decreased between day 0 and day $31(6.45 \pm$ 1.22 vs $3.84 \pm 2.19 ; p<0.0001$ ). More specifically, the median (IQR) of patient's mean number of liquid stools decreased from $6.29(5.1-7.3)$ to $3.7(1.6-6.0)(p=0.0001)$ (Figure 1(a)) while the median (IQR) of patient's mean number of smooth stools did not change significantly $(0(0-0.6)$ vs $0.14(0-0.7) ; p=$ 0.69) (Figure 1(b)).

We also observed a significant decrease of the median (IQR) of patient's mean number of bowel movements, from $6.9(5.6-7.6)$ to $4.6(2.6-6.0)(p<0.0001)$ between day 0 and day 31 (Figure $1(\mathrm{c})$ ). The median (IQR) of the maximum number of bowel movements also significantly decreased, from 9 (7-9) to $5(4-8)(p=0.0009)$ between day 0 and day 31 (Figure $1(\mathrm{~d})$ ).

\section{Safety}

Among the fifteen patients included in the study, only four (26.7\%) presented an adverse event: abdominal pain in 1; nausea and abdominal pain in 2; nausea, abdominal pain and thoracic oppression in 1. None of them presented SAEs.

\section{Other assessments}

Ten out of the 15 patients $(66.7 \%)$ responded to the treatment with a decrease of at least $25 \%$ of liquid or smooth stools per day, and $8 / 15(53.3 \%)$ responded to the treatment with a decrease of at least 2 liquid or smooth stools per day.

We observed a significant decrease of the HBI between day 0 and day 31 , from $9.8+2.6$ to $5.4+4.4$ $(p=0.0006)$. At day 31 , five (33.3\%) patients presented an $\mathrm{HBI}<5$ indicating a remission (Figure 2(a)).

We additionally observed a significant improvement of the quality of life, evaluated by the IBDQ, between baseline and day 31, from $115+25.4$ to $147+37.9(p=0.0012)$ (Figure 2(b)).

There was no significant change in CRP between day 0 and day $31(p=0.26)$ (Figure $3(a))$ and no significant change in fecal calprotectin $(p=0.19)$ (Figure 3(b)).

Table 1. Characteristics of the patients $(N=15)$. 
Published in: Laura Martelli, Arnaud Colard, Fernand Fontaine, Jacques Deflandre, Boris Bastens \& Edouard Louis (2017) Evaluation of the efficacy of octreotide LAR in the treatment of Crohn's disease associated refractory diarrhea, Scandinavian Journal of Gastroenterology, 52:5, 564-569, DOI: 10.1080/00365521.2017.1284893

Status: Postprint (author's version)

\begin{tabular}{|c|c|c|c|c|c|}
\hline Variable & Categories & Number & $\begin{array}{c}\text { Number } \\
(\%)\end{array}$ & Median & $\begin{array}{l}\text { IQR (Q1- } \\
\text { Q3) }\end{array}$ \\
\hline Age (years) & & 15 & & 51.2 & $39.7-58.0$ \\
\hline Sex & $\begin{array}{l}\text { W } \\
M\end{array}$ & 15 & $\begin{array}{l}8(53.3) \\
7(46.7)\end{array}$ & & \\
\hline Disease duration (years) & & 15 & & 14.0 & $5.0-29.0$ \\
\hline Weight (kg) & & 15 & & 72.8 & $62.0-80.4$ \\
\hline Height (m) & & 15 & & 1.72 & 1.7-1.8 \\
\hline $\operatorname{BMI}\left(\mathrm{kg} / \mathrm{m}^{2}\right)$ & & 15 & & 24.1 & $20.9-28.4$ \\
\hline Previous intestinal resection & & 15 & $11(73.3)$ & & \\
\hline Previous cholecystectomy & & 15 & $4(26.7)$ & & \\
\hline Loperamide & & 14 & $5(35.7)$ & & \\
\hline Cholestyramine & & 14 & $2(14.3)$ & & \\
\hline Mesalazine & & 15 & $2(13.3)$ & & \\
\hline Steroids & & 15 & $4(26.7)$ & & \\
\hline Anti-TNF & & 15 & $8(53.3)$ & & \\
\hline Immunosuppressive drugs & & 15 & $7(46.7)$ & & \\
\hline $\mathrm{CRP}(\mathrm{mg} / \mathrm{l})$ at baseline & & 7 & & 3.9 & 0.4-6.7 \\
\hline Fecal calprotectin $(\mu \mathrm{g} / \mathrm{g})$ at baseline & & 14 & & 177 & $32.0-211$ \\
\hline $\mathrm{HBI}$ at baseline & & 11 & & 10.0 & $9.0-12.0$ \\
\hline IBDQ at baseline & & 14 & & 119 & $98.0-135$ \\
\hline $\begin{array}{l}\text { Patients' seven days mean number of stools at } \\
\text { baseline }\end{array}$ & & 15 & & 6.9 & 5.6-7.6 \\
\hline $\begin{array}{l}\text { Patients' seven days mean number of liquid } \\
\text { stools at baseline }\end{array}$ & & 15 & & 6.29 & $5.1-7.3$ \\
\hline $\begin{array}{l}\text { Patients' seven days mean number of smooth } \\
\text { stools at baseline }\end{array}$ & & 15 & & 0.0 & $0.0-0.6$ \\
\hline $\begin{array}{l}\text { Patients' seven days maximum number of } \\
\text { stools at baseline }\end{array}$ & & 15 & & 9.0 & $7.0-9.0$ \\
\hline
\end{tabular}

IQR: Interquartile Range; BMI: Body Mass Index; CRP: C-reactive protein; HBI: Harvey-Bradshaw Index; IBDQ: Inflammatory bowel Disease Questionnaire.

\section{Discussion}

To our knowledge, this is the first study available in the literature evaluating the efficacy and safety of octreotide in CD patients with refractory diarrhea.

Diarrhea is one of the main incapacitating symptoms of CD. It is significantly correlated with an impaired quality of life $[9,10]$. It is therefore one of the major parameters included in various indexes of CD activity and quality of life [7,8,11-14].

For the assessment of the effect of new treatments in CD, the Food and Drug Administration (FDA) is moving from the Crohn's Disease Activity Index (CDAl) [11] to patient-reported outcomes (PROs) and objective measures of the disease, such as findings from endoscopy $[15,16]$. PROs are direct responses from patients about how they feel or function in relation to a health condition and its therapy without interpretation by the physicians [17]. PROs can evaluate symptoms, signs, functional status, perceptions, or other aspects such as convenience and tolerability [17]. PROs will become an important aspect of assessing activity of inflammatory bowel diseases (IBD) [15,16]. Several diseasespecific scales have been developed to assess these PROs and are now commonly used in clinical trials [15]. Outcomes reported by patients in clinical trials of IBD initially focused on quality of life. Recently, considered factors include fatigue, depression, anxiety, and work productivity $[15,18]$. However, few data are available on how treatment affects these factors in patients with IBD.

Figure 1. Evolution of the number of stools between day 0 and day 31. (a) liquid stools, (b) smooth stools, (c) total number of stools, (d) maximum number of stools. 
Published in: Laura Martelli, Arnaud Colard, Fernand Fontaine, Jacques Deflandre, Boris Bastens \& Edouard Louis (2017) Evaluation of the efficacy of octreotide LAR in the treatment of Crohn's disease associated refractory diarrhea, Scandinavian Journal of Gastroenterology, 52:5, 564-569, DOI: 10.1080/00365521.2017.1284893

(a)

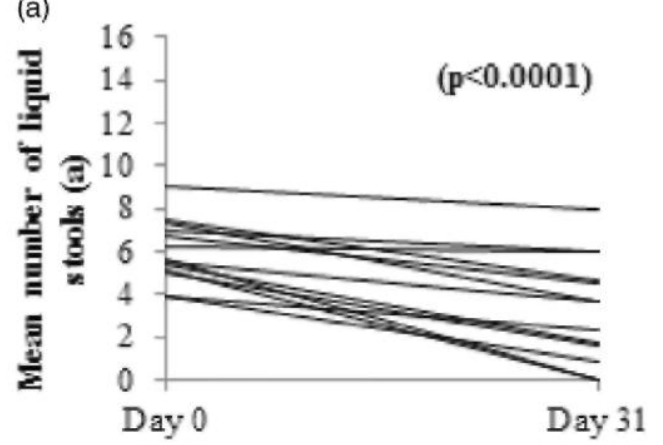

Time

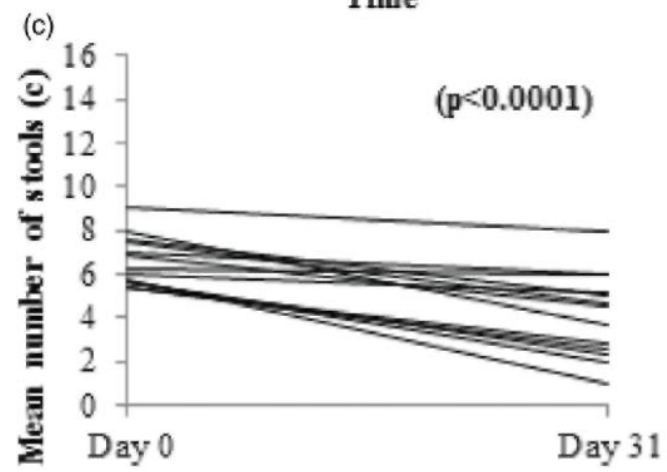

Time

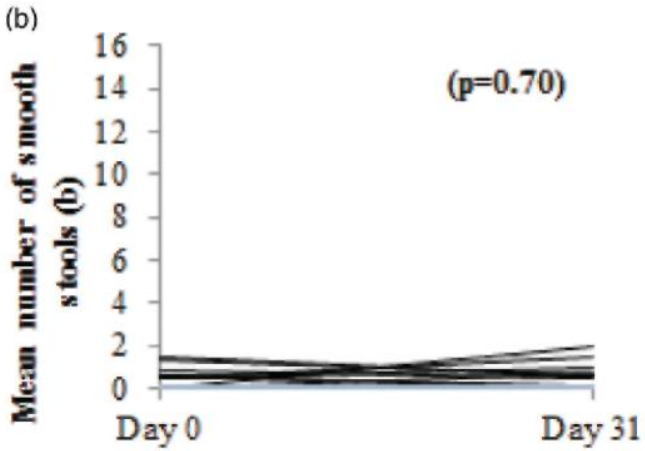

Time

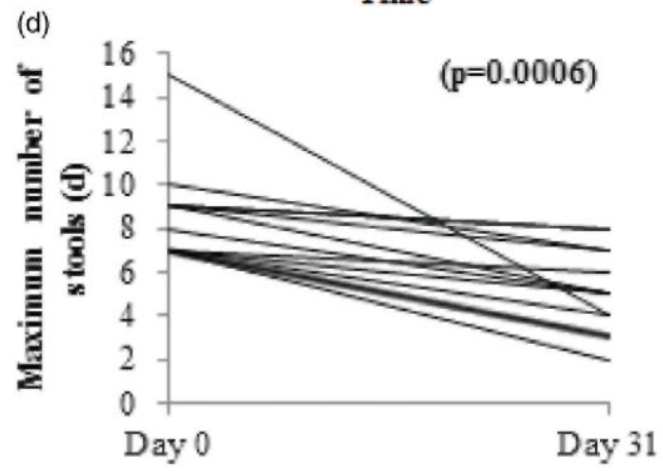

Time

Figure 2. Evolution of $\mathrm{CD}$ activity assessed by the Harvey-Bradshaw Index (HBI) (a) and of quality of life assessed by the IBDQ (b) between day 0 and day 31 .
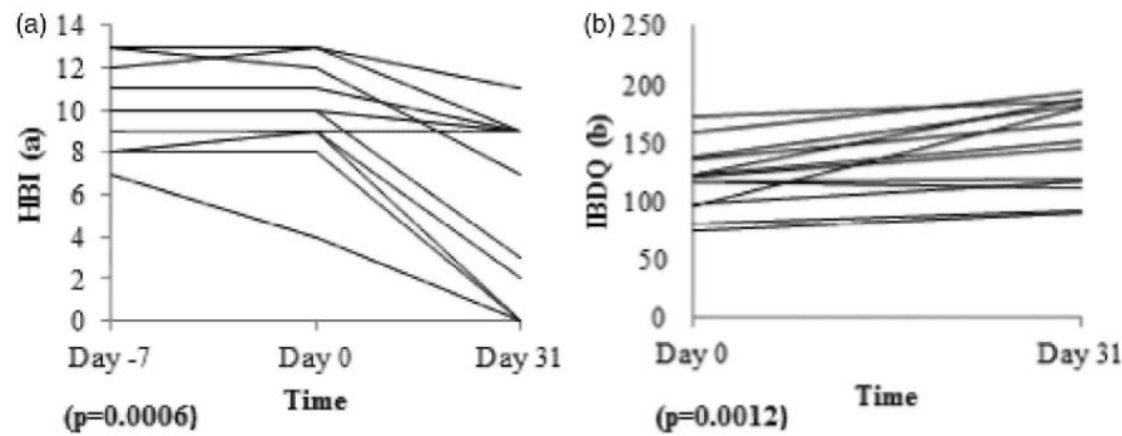

Figure 3. Evolution of biological parameters (CRP measured locally (a), and fecal calprotectin measured centrally (b)) between day 0 and day 31 .
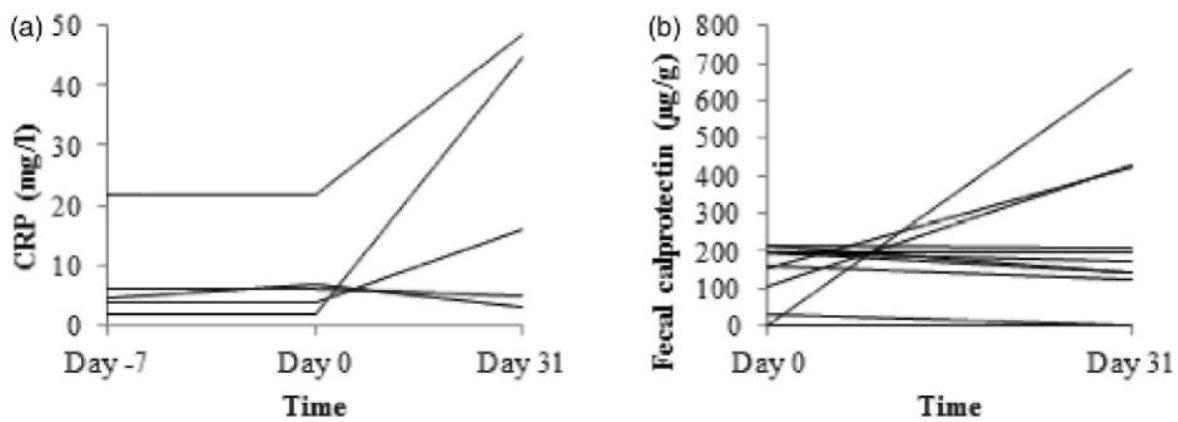
Published in: Laura Martelli, Arnaud Colard, Fernand Fontaine, Jacques Deflandre, Boris Bastens \& Edouard Louis (2017) Evaluation of the efficacy of octreotide LAR in the treatment of Crohn's disease associated refractory diarrhea, Scandinavian Journal of Gastroenterology, 52:5, 564-569, DOI: 10.1080/00365521.2017.1284893

Status: Postprint (author's version)

The mechanisms of diarrhea in CD are complex and multifactorial. Malabsorption, osmotic mechanisms, secretory, and motility anomalies may be involved. Diarrhea is usually significantly improved by specific CD treatments (steroids, immunosuppressive drugs, and biologics). However, in some cases, diarrhea persists despite these treatments and becomes refractory. The mechanisms of this refractory diarrhea can be multiple. It can be related to an incomplete control of the intestinal lesions despite an optimization of CD treatment which is considered maximal. In other cases, despite a complete control of the intestinal lesions, a functional diarrhea may persist. In these situations, loperamide, antibiotics for small bowel overgrowth and cholestyramine may be useful, especially in patients with previous intestinal resection. However, some patients are also resistant to these treatments.

Refractory diarrhea was defined in the present study by at least a mean of five smooth or liquid stools per day (mean of the number of stools per day over the last seven days prior to baseline) despite a normal CRP and an optimized specific CD treatment including immunosuppressive drugs and/or antiTNF. Patients were excluded of the study if they presented diarrhea due to another mechanism (small bowel microbial proliferation, lactose intolerance, irradiation, chemotherapy, neoplasia, or ischemia).

In these situations of CD associated refractory diarrhea, our results suggest a potential efficacy of octreotide. Octreotide can improve diarrhea through different mechanisms: it can have an impact on the motility by prolonging the mouth-to-caecum transit time [1]; it can also have an effect on the gastrointestinal tract secretion [2] by increasing the intestinal absorption in the jejunum and the ileum and reducing the plasma VIP concentrations, the daily stool frequency and the weight of the stools. Through this effect on diarrhea, octreotide also improved $\mathrm{CD}$ activity score $(\mathrm{HBI})$ and health related quality of life (IBDQ).

Octreotide can also have a potential immunomodulatory effect [4] by interacting directly with its specific receptors that are expressed on the intestinal epithelial cells. It can down-regulate proinflammatory mediators secretion by a mechanism involving the regulation of transcription. These findings suggest that somatostatin and its analogs may play an active role in regulating the mucosal inflammatory response of intestinal epithelial cells after physiological and pathological stimulations such as bacterial invasion. However, our results do not suggest any significant effect of this kind since there was no significant change in CRP and fecal calprotectin.

The safety profile of octreotide was also favorable without any SAEs and only mild nonspecific AEs reported by a minority of patients. These results are similar to those published in the literature $[19,20]$.

\section{Conclusions}

In this uncontrolled open-label study, octreotide appeared safe and efficacious in patients with CD refractory diarrhea, in addition to specific CD treatments. It significantly improved the number of liquid or smooth stools, the $\mathrm{HBI}$ and the IBDQ.

\section{Disclosure statement}

The authors report no conflicts of interest.

\section{Funding}

Financial support for the present study was provided by Novartis Belgium. Novartis had no impact on the design of the study, on the interpretation of the results and the writing of the paper.

\section{References}

[1] O'Donnell U, Watson AJ, Cameron D, et al. Effect of octreotide on mouth-to-caecum transit time in healthy subjects and in the irritable bowel syndrome. Aliment Pharmacol Ther. 1990; 4:177-181.

[2] Edwards C, Cann PA, Read NW, et al. Effect of two new antisecretory drugs on fluid and electrolyte transport in a patient with secretory diarrhoea. Gut. 1986;27:581-586.

[3] Cooper JC, Williams NS, King RF, et al. Effects of a long-acting somatostatin analogue in patients with severe ileostomy diarrhoea. Br J Surg. 1986;73:128-131.

[4] Chowers Y, Cahalon L, Lahav M, et al. Somatostatin through its specific receptor inhibits spontaneous and TNF-alpha and bacteria-induced IL-8 and IL-1 beta secretion from intestinal epithelial cells. J Immunol. 2000;165:2955-2961.

[5] Lennard-Jones JE. Classification of inflammatory bowel disease. Scand J Gastroenterol Suppl. 1989;170:2-6.

[6] Lewis SJ, Heaton KW. Stool form scale as a useful guide to intestinal transit time. Scand J Gastroenterol. 1997;32:920-924.

[7] Harvey RF, Bradshaw JM. A simple index of Crohn's-disease activity. Lancet. 1980;1:514. 
Published in: Laura Martelli, Arnaud Colard, Fernand Fontaine, Jacques Deflandre, Boris Bastens \& Edouard Louis (2017) Evaluation of the efficacy of octreotide LAR in the treatment of Crohn's disease associated refractory diarrhea, Scandinavian Journal of Gastroenterology, 52:5, 564-569, DOI: 10.1080/00365521.2017.1284893

Status: Postprint (author's version)

[8] Guyatt G, Mitchell A, Irvine EJ, et al. A new measure of health status for clinical trials in inflammatory bowel disease. Gastroenterology. 1989;96:804-810.

[9] Ghosh S, Mitchell R. Impact of inflammatory bowel disease on quality of life: results of the European Federation of Crohn's and Ulcerative Colitis Associations (EFCCA) patient survey. J Crohns Colitis. 2007;1:10-20.

[10] Lonnfors S, Vermeire S, Greco M, et al. IBD and health-related quality of life - discovering the true impact. J Crohns Colitis. 2014;8:1281-1286.

[11] Best WR, Becktel JM, Singleton JW, et al. Development of a Crohn's disease activity index. National Cooperative Crohn's Disease Study. Gastroenterology. 1976;70:439-444.

[12] Irvine EJ, Zhou Q, Thompson AK. The short inflammatory bowel disease questionnaire: a quality of life instrument for community physicians managing inflammatory bowel disease. Am J Gastroenterol. 1996;91:1571 -1578.

[13] Thia K, Faubion WA, Jr, Loftus EV, Jr, et al. Short CDAl: development and validation of a shortened and simplified Crohn's disease activity index. Inflamm Bowel Dis. 2011;17:105-111.

[14] Alrubaiy L, Cheung WY, Dodds P, et al. Development of a short questionnaire to assess the quality of life in Crohn's disease and ulcerative colitis. J Crohns Colitis. 2015;9:66-76.

[15] Williet N, Sandborn WJ, Peyrin-Biroulet L. Patient-reported outcomes as primary end points in clinical trials of inflammatory bowel disease. Clin Gastroenterol Hepatol. 2014;12:1246-1256.

[16] Khanna R, Zou G, D'Haens G, et al. A retrospective analysis: the development of patient reported outcome measures for the assessment of Crohn's disease activity. Aliment Pharmacol Ther. 2015;41:77-86.

[17] Kappelman MD, Long MD, Martin C, et al. Evaluation of the patient reported outcomes measurement information system in a large cohort of patients with inflammatory bowel diseases. Clin Gastroenterol Hepatol. 2014;12:1315-1323.

[18] Gater A, Kitchen H, Heron L, et al. Development of a conceptual model evaluating the humanistic and economic burden of Crohn's disease: implications for patient-reported outcomes Measurement and economic evaluation. Expert Rev Pharmacoecon Outcomes Res. 2015;15:643-656.

[19] Wass JA, Popovic V, Chayvialle JA. Proceedings of the discussion,"Tolerability and safety of Sandostatin". Metab Clin Exp.1992;41:80-82.

[20] Bornschein J, Drozdov I, Malfertheiner P. Octreotide LAR: safety and tolerability issues. Expert Opin Drug Saf. 2009;8:755768. 